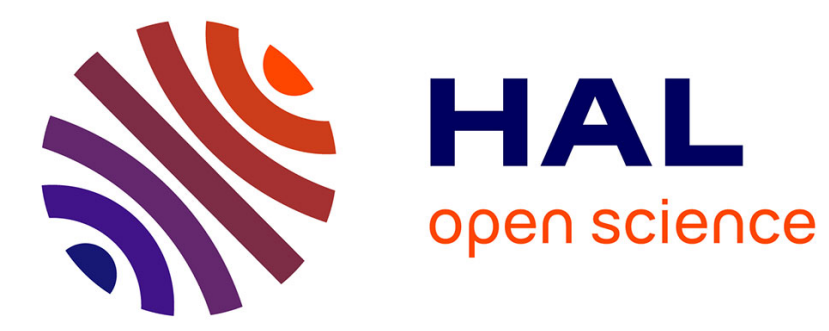

\title{
Highly cohesive dual nanoassemblies for complementary multiscale bioimaging
}

Adrien Faucon, Thomas Maldiney, Olivier Clément, Philippe Hulin, Steven

Nedellec, Myriam Robard, Nicolas Gautier, Evelien de Meulenaere, Koen

Clays, Tomas Orlando, et al.

\section{To cite this version:}

Adrien Faucon, Thomas Maldiney, Olivier Clément, Philippe Hulin, Steven Nedellec, et al.. Highly cohesive dual nanoassemblies for complementary multiscale bioimaging. Journal of materials chemistry B, 2014, 2, pp.7747 - 7755. 10.1039/C4TB01199F . cea-01376834

\section{HAL Id: cea-01376834 https://hal-cea.archives-ouvertes.fr/cea-01376834}

Submitted on 5 Oct 2016

HAL is a multi-disciplinary open access archive for the deposit and dissemination of scientific research documents, whether they are published or not. The documents may come from teaching and research institutions in France or abroad, or from public or private research centers.
L'archive ouverte pluridisciplinaire HAL, est destinée au dépôt et à la diffusion de documents scientifiques de niveau recherche, publiés ou non, émanant des établissements d'enseignement et de recherche français ou étrangers, des laboratoires publics ou privés. 


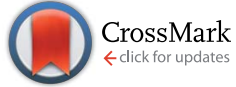

Cite this: J. Mater. Chem. B, 2014, 2 , 7747

Received 20th July 2014

Accepted 11th September 2014

DOI: 10.1039/c4tb01199f

www.rsc.org/MaterialsB

\section{Highly cohesive dual nanoassemblies for complementary multiscale bioimaging $\dagger$}

\author{
Adrien Faucon, ${ }^{a}$ Thomas Maldiney, ${ }^{\mathrm{b}}$ Olivier Clément, ${ }^{\mathrm{b}}$ Philippe Hulin, ${ }^{\mathrm{c}}$ \\ Steven Nedellec, ${ }^{c}$ Myriam Robard, ${ }^{c}$ Nicolas Gautier, ${ }^{d}$ Evelien De Meulenaere, ${ }^{\text {ef }}$ \\ Koen Clays, ${ }^{\text {e }}$ Tomas Orlando, ${ }^{g}$ Alessandro Lascialfari, ${ }^{\text {h }}$ Céline Fiorini-Debuisschert, \\ Jérôme Fresnais ${ }^{j}$ and Eléna Ishow*a
}

\begin{abstract}
Innovative nanostructures made of a high payload of fluorophores and superparamagnetic nanoparticles (NPs) have simply been fabricated upon self-assembling in a two-step process. The resulting hybrid supraparticles displayed a dense shell of iron oxide nanoparticles tightly attached through an appropriate polyelectrolyte to a highly emissive non-doped nanocore made of more than $10^{5}$ small organic molecules. Cooperative magnetic dipole interactions arose due to the closely packed magnetic NPs at the nanoarchitecture surface, causing enhanced NMR transverse relaxivity. Large in vivo MRI $T_{2}$ contrast was thus obtained with unusually diluted solutions after intravenous injection in small rodents. Twophoton excited fluorescence imaging could be performed, achieving unprecedented location resolution for agents combining both magnetic nanoparticles and fluorescence properties. Finally, TEM imaging of the sectioned mouse tissue succeeded in isolating the core-shell structures, which represents the first image of intact complex magnetic and fluorescent nanoassemblies upon in vivo injection. Such highly cohesive dual nanoarchitectures should open great horizons toward the assessment with high spatial resolution of the drug or labeled stem cell biodistribution.
\end{abstract}

\section{Introduction}

Multifunctional nanomaterials have recently emerged as promising contrast agents for in vitro and in vivo bioimaging. ${ }^{1-6}$ Compared to their dispersed molecular fluorescent probes ${ }^{7,8}$ and superparamagnetic complexes, ${ }^{\mathbf{9}, 10}$ they exhibit greater density of active units and longer body circulation time. ${ }^{\mathbf{1 1}}$

${ }^{a}$ CEISAM - UMR CNRS 6230, Université de Nantes, 2 rue de la Houssinière, 44322 Nantes, France. E-mail: elena.ishow@univ-nantes.fr

${ }^{b}$ Paris Cardiovascular Research Center - PARCC, Université Paris Descartes, INSERM U970, 20 rue Leblanc, 75015 Paris, France

'INSERM UMS 016-UMS CNRS 3556, 8 quai Moncousu, 44007 Nantes, France ${ }^{d} I M N$ - UMR CNRS 6502, Université de Nantes, 2 rue de la Houssinière, 44322 Nantes, France

${ }^{e}$ Department of Chemistry, KULeuven, Celestijnelaan 200D, 3001 Heverlee, Belgium ${ }^{f}$ Department of Bioscience Engineering, KULeuven, Kasteelpark Arenberg 20, 3001 Heverlee, Belgium

${ }^{g}$ Department of Physics and INSTM, Università di Pavia, via Bassi, 27100 Pavia, Italy ${ }^{h}$ Department of Physics and INSTM, Università degli Studi di Milano, via Celoria 16, 20133 Milano, Italy

${ }^{i}$ CEA-Saclay, DSM-IRAMIS-SPEC, 91191 Gif-sur-Yvette, France

${ }^{j}$ PECSA - UMR CNRS 7195, Université Pierre et Marie Curie, 4 place Jussieu, 75005 Paris, France

$\dagger$ Electronic supplementary information (ESI) available: Nanoassembly colloidal stability in various media, two-photon spectral detection and fluorescence lifetime imaging of sections of the mouse liver after in vivo injection, and the protocol of tissue sectioning and staining. See DOI: 10.1039/c4tb01199f
Hence, the injected doses are dramatically reduced and the kinetic follow-up is prolonged. The combination of fluorescence $^{12}$ and magnetism ${ }^{13}$ has appeared as particularly attractive since tunable remote excitation is performed in both cases, and cross-correlations between in vivo and histology studies are possible. ${ }^{\mathbf{1 4 , 1 5}}$ While fluorescence imaging offers high sensitivity and spatial resolution due to the outstanding performance of optical detectors, magnetic resonance imaging encounters no tissue penetration limits. Much effort has been devoted to the grafting of molecular luminophores onto iron oxide superparamagnetic nanoparticles (NPs) ${ }^{\mathbf{1 6}, 17}$ or to the dual encapsulation of both emissive and magnetic entities in biocompatible matrices. ${ }^{15,18-20}$ However, the latter often face a low fluorescence signal due to physical dilution and emission quenching by the components themselves or the surroundings. In this context, we devised a disruptive approach involving an organic functional platform, ${ }^{21}$ made of small fluorescent molecules amenable to spontaneously self-assemble without losing their emission signal in rigid nanospheres. The resulting organic fluorescent nanoparticles (FON) spontaneously chelated maghemite $\gamma$ $\mathrm{Fe}_{2} \mathrm{O}_{3}$ nanoparticles at their surface in aqueous media, avoiding the accumulation of a high amount of organic solvent. ${ }^{22}$ This simple one-step procedure generated innovative nanoarchitectures fluo@mag exhibiting a "raspberry-like" structure and revealing a single-photon brightness of around $10^{7} \mathrm{~mol}^{-1} \mathrm{~L}$ $\mathrm{cm}^{-1}$, comparable to that of quantum $\operatorname{dots}^{23}$ or polymer 
dots, ${ }^{24,25}$ thanks to the high payload $\left(>10^{5}\right.$ molecules per nanoassembly) of functional units usually reported for FON. ${ }^{26-28}$ As the fluorophores are tightly packed with each other through hydrophobic interactions and coated with a protective shell of charged nanoparticles, the risks of dye leakage or enzymatic cleavage are strongly reduced compared to those commonly observed for architectures doped with or functionalized at their surface with fluorophores. Finally the fluo@mag nanoassembly emission, originating from the protected fluorescent core, was not subjected to photoblinking, and advantageously found insensitive to the surroundings in contrast with the latter semiconducting nanomaterials, making their follow-up over time possible. Yet, their preparation in diluted nitric acid precluded further biological applications. Moreover after some time, disassembling of the structure occurred due to the presence of oxidative nitrate ions. In this work, we want to show that very stable colloidal solutions, compatible with biological media of various $\mathrm{pH}$ and ionic strengths, have been obtained using an appropriate polylelectrolyte coating. Compared to numerous architectures like polymersomes or liposomes, the stabilized nanoassemblies display very high structural cohesion after in vivo injection. The high density of magnetic nanoparticles leads to cooperative dipole effects, so that straightforward comparative investigations at various scales can be achieved using twophoton excited fluorescence imaging and in vivo magnetic resonance imaging (MRI).

\section{Results and discussion}

\section{Nanoparticle assembling process and structural characterizations}

A typical procedure to generate stable bimodal nanoassemblies consisted of the quick injection of a water-miscible concentrated stock solution of fluorescent phosphonic acid compound into an acidic solution of maghemite NPs (Scheme 1). The use of nitric acid ensures stable iron oxide nanoparticles as nitrate ions are non-coordinating anions conversely to chloride ones.

Fast dye nanoprecipitation occurred, forming FON that spontaneously complexed a dense shell of iron oxide NPs due to the presence of phosphonic units. Addition of polyacrylic acid (PAA), known for its biocompatibility and good affinity to iron, was performed in the second step to bring high cohesion to the self-assembled architecture fluo@mag. The pH solution was adjusted by adding a $1 \mathrm{~mol} \mathrm{~L}^{-1}$ ammonium hydroxide solution until $\mathrm{pH}=9$, to ensure extensive PAA deprotonation $\left(\mathrm{p} K_{\mathrm{a}}=6.8\right)$ and efficient coating of maghemite NPs. The excess polymer was finally removed by dialysis against Millipore water. The dialysis step appears also useful to yield nanoassemblies with more regular surface when compared to those obtained directly after precipitation, due to probable rearrangements occurring at the complexing interface. Keeping the polymer chain short enough $(<30 \mathrm{kDa})$ appeared crucial to avoid irreversible clustering of fluo@mag NPs. We thus privileged $2.1 \mathrm{kDa}$ PAA that gave the best results. No such stabilization was obtained with small chelating ions like citrate, due to fast ligand exchange equilibrium, leading in turn to the progressive loss of iron NPs. Interestingly, self-assembly of magnetic nanoparticles using an amphiphilic polyelectrolyte and a solvent-shifting process have recently been reported..$^{29,30}$ The aggregation process yields dense magnetic assemblies with quite narrow size dispersion. However, to the best of our knowledge, no studies so far have already reported the use of functional amphiphilic small molecules as mentioned herein, to fabricate bimodal architectures following a simple one-step procedure. The current approach considerably simplifies the purification process as dyes and any trace of organic solvent can easily be eliminated by magnetic decantation or dialysis using small-pore membranes. Moreover, assembling small molecules, largely used in photonics, ${ }^{27,31-34}$ also allows the direct incorporation of electro-, photo- or $\mathrm{pH}$-active moieties to impart the nanoassemblies with functional activities without requiring additional cumbersome modification of the nanoparticle surface.

Size measurements of the fluo@mag@PAA nanoassemblies by dynamic light scattering (DLS) showed a $190 \mathrm{~nm}$ mean hydrodynamic diameter $D_{\mathrm{H}}$. This value is substantially larger than the $150 \mathrm{~nm}$ mean diameter obtained by transmission electron microscopy (TEM), due to the large hydration shell promoted by the PAA coating (Fig. 1). ${ }^{35}$

The nanoassemblies exhibit a highly negative surface potential $(\zeta=-31 \mathrm{mV})$ as measured by zetametry, ensuring long-term stability with no size change over months at $4{ }^{\circ} \mathrm{C}$. Lyophilization could advantageously be performed, which enables further clean nanoassembly redispersion in various media like ethanol, PBS or HBSS cell culture buffer, and physiological serum. DLS measurements revealed no sign of aggregation compared to the initial solution (Fig. 2a).

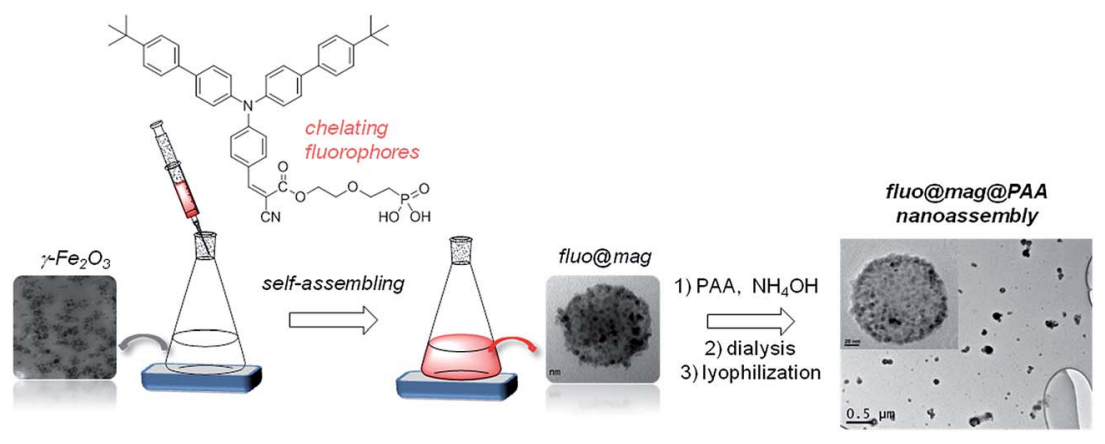

Scheme 1 Fabrication procedure of colloidally stable fluo amag@PAA nanoassemblies in aqueous solutions. 


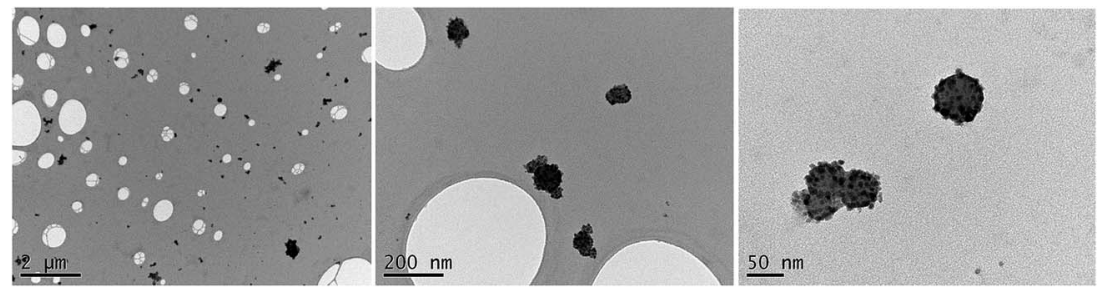

Fig. 1 TEM imaging of fluoamagaPAA nanoassemblies deposited on holey carbon coated copper grids.

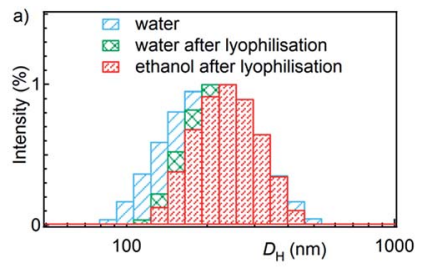

100
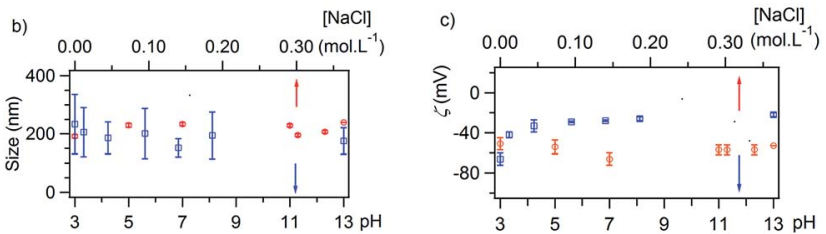

Fig. 2 DLS measurements of the colloidal suspensions (a) before lyophilization and after redispersion in various media. (b) DLS size measurements and (c) surface potential $\zeta$ measurements as a function of the solution $\mathrm{pH}$ and ionic strength.

UV-vis absorption measurements, recorded over a period of $14 \mathrm{~h}$ at day 1, 6 and 11 after redispersion of fluo@mag@PAA nanoassemblies in HBSS buffer, showed no significant absorbance loss (see ESI Fig. S1 $\dagger$ ). Large agglomerates would have led instead to decreasing absorption due to fast decantation, which is not observed as absorbance changes by $1 \%$ only. Handling solid nanomaterials to prepare solutions right before each targeted application is actually of prime importance since solution concentrations can precisely be controlled with no risk of drug hydrolysis or solvent evaporation. Colloidal stabilities were also checked over a wide range of $\mathrm{pH}$ and ionic strengths. The fluo@mag@PAA nanoassemblies exhibit again no aggregation in aqueous solutions of $\mathrm{pH}=3-12$ and ionic strength up to 0.3 mol L ${ }^{-1}$ of $\mathrm{NaCl}$ (Fig. $2 \mathrm{~b}$ and c) as no significant changes in size or surface potential were noted. Such stabilities are very crucial for any in vivo applications as clustering would lead to functional deactivation or risks of embolism upon vessel clogging.

Quantification of the averaged number of iron oxide nanoparticles immobilized on the nanoassemblies has been performed by resorting to inductively coupled plasma optical emission spectrometry (ICP-OES) titrations, following acidic mineralization. Analyses using iron standard solutions provided a coating of around 5000 maghemite NPs per nanoassembly (see ESI $\dagger$ for calculations). This number suggests the presence of more than one layer of iron oxide NPs since a stiff monolayer of maghemite NPs would give a surface coverage 5-7 times less dense.

\section{Magnetic resonance relaxivities}

The ability of fluo@mag@PAA nanoassemblies to act as MRI contrast agents was assessed by measuring the proton transverse $r_{2}$ and longitudinal $r_{1}$ magnetic resonance (MR) relaxivities. The latter were determined by varying the Larmor proton frequencies from 0.01 to $60 \mathrm{MHz}$, covering a range of magnetic fields commonly used in clinical MRI tomographs $(f=8.5,21$, $64 \mathrm{MHz}$, corresponding to an applied magnetic field $H=0.2,0.5$ and $1.5 \mathrm{~T})$. Longitudinal relaxivity $\left(r_{1}\right)$ follows the typical behaviour of superparamagnetic nanoparticles, where one can distinguish: a low frequency plateau, due to the reorientation of the single particle magnetization (Néel relaxation), a rapid decrease at high frequencies (Curie relaxation) and a maximum around $5 \mathrm{MHz}$, due to the superposition of these two mechanisms. Transverse relaxivity $r_{2}$ is of special interest in our case since it features the efficiency of the MR image contrast of superparamagnetic negative contrast agents. For frequencies larger than $1 \mathrm{MHz}$, the $r_{2}$ value for fluo@mag@PAA was found to be almost twice as high as that for free oxide NPs stabilized by PAA in neutral water (mag@PAA) (Fig. 3a). Such an increase in $r_{2}$, up to $238 \pm 19 \mathrm{~s}^{-1} \mathrm{mmol}^{-1} \mathrm{~L}$, is probably due to the total magnetization of the nanostructure, to which $r_{2}$ is strongly related..$^{28}$ Indeed, the tight packing of iron oxide nanoparticles at the surface of the fluorescent core brings them in close vicinity, which causes cooperativity effects. This generates an increase of the total magnetization of the single nanostructure (fluo@mag@PAA), that is approximately the sum of the magnetizations of the iron oxide nanoparticles at high fields.

Even more noticeable, the ratio $r_{2} / r_{1}$ (the higher this parameter, the shorter the acquisition times for similar image contrasts) reaches a maximum of 10 at $20 \mathrm{MHz}(0.5 \mathrm{~T})$, which is 2.5 times larger than that of the commercially available
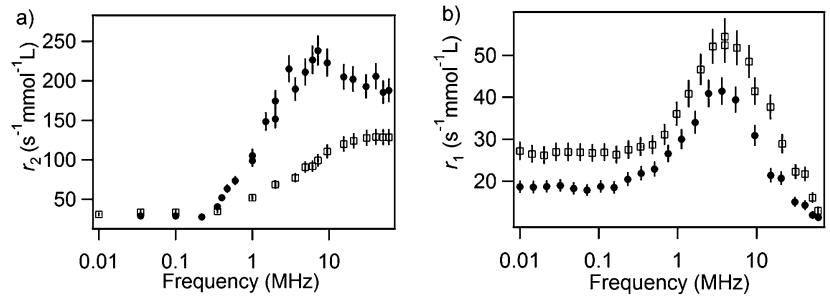

Fig. 3 (a) Transverse $r_{2}$ and (b) longitudinal $r_{1}$ relaxivities of fluoa mag (APAA nanoassemblies ( $D_{\mathrm{H}}=150 \mathrm{~nm}$; black dots) and dispersed magaPAA ( $D_{\mathrm{H}}=7 \mathrm{~nm}$; blank squares), measured at $25^{\circ} \mathrm{C}$. $D_{\mathrm{H}}$ features the hydrodynamic diameter. 
Endorem $T_{2}$ contrast agent, measured under identical experimental conditions. ${ }^{36}$ By contrast, $r_{2} / r_{1}$ changed only from 1.4 to 4.2 for the PAA-coated maghemite NPs mag@PAA. Synergistic dipole-dipole interactions between the tightly connected magnetic NPs at the nanoassembly surface are undoubtedly responsible for the two- to threefold $r_{2} / r_{1}$ increase, as already noted for various ensembles of nanoparticles packed within the same supraparticle. ${ }^{29,37-39}$ This novel approach thus represents an attractive and simple way to increase substantially the magnetic response and decrease the injected dose as highly requested for in vivo applications. These encouraging results prompted us to undergo in vivo MRI experiments and test the performances of fluo@mag@PAA nanoassemblies as $T_{2}$ dark contrast agents.

\section{MRI in small rodents}

Prior to in vivo injection, we checked the cell viability of two common cell lines (MDA-MB-468, HEK-293) in the presence of fluo@mag@PAA. Various viability controls (nanoassembly incubation for $60 \mathrm{~h}$, or incubation for 6-8 h followed by a further $60 \mathrm{~h}$ chase incubation step) using MTT (3-[4,5-dimethylthiazol-2-yl]-2,5-diphenyl tetrazolium bromide) were performed. They reveal almost no cell cytotoxicity (cell viability $>95 \%$ ) for nanoassembly suspensions with a $15 \mu \mathrm{mol} \mathrm{L}{ }^{-1}$ iron content that is the common concentration used for all the in vitro and in vivo experiments (live cell studies represent entire studies per se that are beyond the scope of this manuscript as this kind of reverse architectures had never been studied in vitro so far).

MRI potentialities of the nanoassemblies were then tested on a population of five female BALB/c mice from the same litter. The mice were imaged at pre-injection and $30 \mathrm{~min}$ post-injection of HBSS buffer suspensions of nanoassemblies $(250 \mu \mathrm{L}$, $1.30 \mu \mathrm{mol} \mathrm{mL}{ }^{-1}$ iron - namely $0.73 \mathrm{mg}$ iron per kg mouse) into the caudal vein. Given the size of the nanoassemblies, the absence of poly(ethylene glycol) coating and the global negatively surface charge, clearance of the nanoassemblies from circulation is rapidly accomplished by the mononuclear phagocyte system (reticuloendothelial system), mostly within the liver and spleen. Superparamagnetic NPs create strong inhomogeneity of the local magnetic field sensed by water protons. Since this "susceptibility effect" causes accelerated proton spin dephasing and faster transverse magnetization relaxation, we adopted a $T_{2}{ }^{*}$-weighted gradient echo sequence at $4.7 \mathrm{~T}$. The retained echo time $\mathrm{TE}=5 \mathrm{~ms}$ and response time $\mathrm{TR}=300 \mathrm{~ms}$ were adjusted to unusually low values to visualize the hypointense area due to the presence of $T_{2}$ contrast agents, and recall those used for highly confined and positively interacting iron oxide nanoparticles. ${ }^{\mathbf{1 5 , 4 0}}$ Averaged dark contrast MRI enhancements (ENH) of $-49 \%$ and $-13 \%$ were found for the liver and spleen, respectively (Fig. 4).

These values, comparable to those in the literature, are very remarkable as the iron dose used herein is almost 10 times lower than those commonly utilized, reducing the risks of cytotoxicity and inflammatory side-reactions. These results are to be related to the very cohesive structure and consequently the
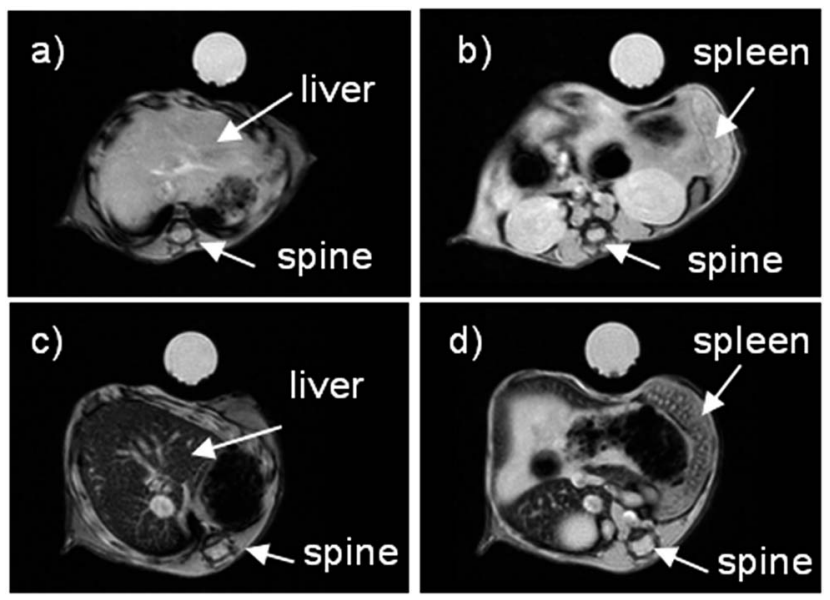

Fig. $4 T_{2}{ }^{*}$-weighted gradient echo (TE $=5 \mathrm{~ms}$, TR $=300 \mathrm{~ms}$ ) MR images of the mouse liver (left) and spleen (right). (a) and (b) Before injection; (c) and (d) at 30 min post-injection The white circle corresponds to a tube filled with water serving as a MRI phantom.

cooperative magnetic interactions induced upon assembling a high payload of maghemite nanoparticles. They fully correlate with the enhancement in solution of the $r_{2}$ transverse relaxivity and the $r_{2} / r_{1}$ ratio as reported above.

One- and two-photon excited fluorescence microscopy imaging

We exploited the fluorescence modality of the fluo@mag@PAA nanoassemblies to check the nanoassembly structural integrity after in vivo injection and their possible localization in other organs. The UV-vis absorption spectrum display both contributions of the maghemite (high energy tail) and the FON core (450 $\mathrm{nm}$ centered transition). Remarkably, no significant change in the emission energy $\left(\lambda_{\mathrm{em}}=604 \mathrm{~nm}\right)$ and emission quantum yield $\Phi_{\mathrm{f}}\left(\Phi_{\mathrm{f}}=0.01\right)$ was noticed between FON and fluo@mag@PAA (Fig. 5). This is due first to the fluorophore embedment in the core, limiting emission deactivation upon electron transfer to the surrounding iron oxide nanoparticles, and secondly to the weak spectral overlap between the

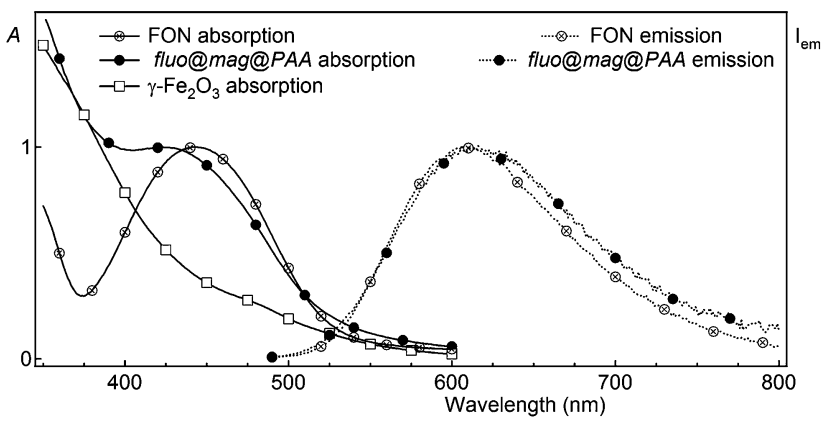

Fig. 5 UV-vis absorption spectra in water of $\mathrm{FON}$ and $\gamma-\mathrm{Fe}_{2} \mathrm{O}_{3}$ nanoparticles, and emission spectra ( $\ddot{e}_{\text {exc }}=450 \mathrm{~nm}$ ) of FON and fluo@mag@PAA nanoassemblies. 
fluorophore emission band and the maghemite absorption one, avoiding an undesirable inner filter effect.

Since liver is a strongly pigmented organ giving rise to high autofluorescence, two-photon fluorescence microscopy imaging, allowing excitation in the tissue transparency window [700-1100 nm], would represent an attractive tool to identify the nanoassemblies. To this aim, we measured the two-photon absorption properties of fluo@mag@PAA by using the twophoton excited fluorescence (TPEF) method. ${ }^{41}$ This technique relies on the comparative measurements of the two-photon induced fluorescence signal of both the sample and the reference (in our case, fluorescein in $0.1 \mathrm{~mol} \mathrm{~L}^{-1} \mathrm{NaOH}$ solution). The two-photon absorption (TPA) maximum for fluo@ mag@PAA appeared at $920 \mathrm{~nm}$, namely at around half of the one-photon absorption energy as expected from the dipolar character of the fluorophore, breaking the symmetry selection rules of electric dipole transitions. The corresponding value of the TPA cross section $\sigma_{\text {TPA }}$ was calculated at $75 \mathrm{GM}$, in agreement with the TPA values previously obtained for FON made of similar fluorescent push-pull triarylamines. ${ }^{42}$ From prior photothermal studies, performed on fluorescent organic nanoparticles, ${ }^{43}$ we could infer that about $N=5.2 \times 10^{5}$ molecules were comprised into the $120 \mathrm{~nm}$ fluorescent core. This allowed us to calculate the global two-photon brightness defined as $N \sigma_{\text {TPA }} \Phi_{\mathrm{f}}$. We found a large value of $3.9 \times 10^{5} \mathrm{GM}$, showing a clear advantage in terms of easy detection of FON-confined dyes over isolated molecular probes, due to their high molecular density, despite modest $\Phi_{\mathrm{f} \cdot{ }^{\mathbf{4 4 , 4 5}}}$

In order to visualize the nanoparticles injected in vivo, the mice were euthanized $24 \mathrm{~h}$ after injection. Their main organs (liver, spleen, kidney, lung and heart) were harvested, fixed with care to avoid dissolution or degradation of the organic fluorophores, and frozen in water-soluble glycols and resin. All tissues were sectioned at $-20{ }^{\circ} \mathrm{C}$ in $10 \mu \mathrm{m}$ thick slices and cell nuclei were counterstained with DAPI $\left(4^{\prime}, 6^{\prime}\right.$-diamido-2-phenylindole). Fluorescence confocal laser scanning microscopy (LSM), equipped with a multichannel GaAsP detector, was performed at $830 \mathrm{~nm}$ on organs extracted from the same mouse. Dispersed bright spots, emitting at $575 \mathrm{~nm}$, could again clearly be distinguished from the strong liver auto-fluorescence (Fig. 6 - see ESI Fig. S2†).

As expected from the 100-200 $\mathrm{nm}$ size range of the nanoassemblies, their amount is significantly higher in liver than in spleen. Only one nanoassembly could be found in the investigated kidney slice while none of them was retained in the lungs or the heart (see ESI Fig. S3-S5†). Z-scan imaging using twochannel detection centered at $450 \mathrm{~nm}$ for the DAPI emission and a wide window of 550-758 $\mathrm{nm}$ for the nanoassemblies evidenced that the nanoassemblies did not penetrate into the nuclei (Fig. 6).

The observation of well-defined emissive spots clearly proves that the nanoassemblies have not been disintegrated upon in vivo interactions with the phagocytic cells. Indeed, comparative studies have been performed on several cell lines with various FON and fluo@mag@PAA nanoassemblies. The former fluorescent nanoparticles, devoid of magnetic coating, dissolved in their individual components, leading to a diffused green-shifted


Fig. 6 TPEF laser scanning image of the mouse liver (left) and spleen (right) sections ( $\lambda_{\text {exc }}=830 \mathrm{~nm}$ ). Top: 2D-spectral single plane images, with lambda-coded coloring, giving the most prominent spectral properties of each pixel. The DAPI-stained nuclei appear blue, the fluoamag@PAA nanoassemblies appear as yellow-orange spots, indicating a fluorescence maximum around $600 \mathrm{~nm}$. Bottom: 3D reconstruction of $10 \mu \mathrm{m}$ thick slices in two-channel detection mode showing DAPI-stained nuclei in blue (411-490 nm) and fluoa mag@PAA nanoassemblies in red (550-758 nm).

signal in the cytoplasm along with localized spots, as already demonstrated in previous experiments, performed on similar self-assembling dyes. ${ }^{46}$ The additional presence of PAA as an external coating undoubtedly reinforced the overall structural cohesion. No such visualization could be done with free molecular probes that would be cleared from the body circulation within a few minutes.

Although TPEF imaging clearly achieves deeper light penetration and a larger signal-to-noise ratio, one-photon excitation at $488 \mathrm{~nm}$ also shows bright spots discernible from the autofluorescent liver background (see ESI Fig. S6†). Spectral detection combined with fluorescence lifetime imaging $\left(\lambda_{\text {exc }}=485\right.$ $\mathrm{nm}$ ) rules out any contribution from lipofuscin aggregates all the more since injection was performed in young mice where lipofuscin yield is low (see ESI Fig. S7†). ${ }^{47}$ Such straightforward assignment of the nanoassembly actually stems not only from the high density of active units comprised in the fluorescent core, but also from the large Stokes shift, up to $5500 \mathrm{~cm}^{-1}$, avoiding emission reabsorption. To the best of our knowledge, this is the first time that the location of emissive organic dyes inside highly pigmented tissues like the liver could be unambiguously imaged using optical fluorescence microscopy. Most of the studies indeed visualize the entire excised organs to eliminate the strong autofluorescence of the biological material and gain a higher signal-to-noise ratio, which however could not reveal defined distribution of accumulated dyes.

\section{TEM imaging of sectioned liver}

In order to gain a scale factor in the nanoassembly localization, we attempted to visualize by TEM the nanoassembly in liver 


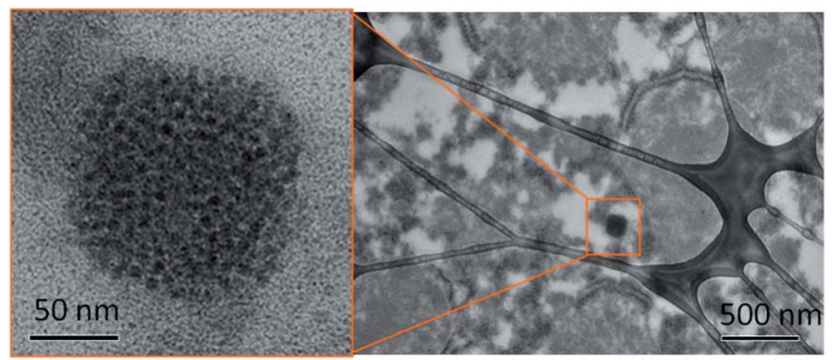

Fig. 7 TEM image of a $70 \mathrm{~nm}$ thin slice of the mouse liver deposited on a holey coated carbon grid $(\times 9 \mathrm{k})$. Inlet: magnification $\times 73 \mathrm{k}$.

sections, based on the clear electronic contrast provided by the iron oxide nanoparticles. To maintain the liver integrity, the entire livers were slowly thawed and fixed in glutaraldehyde over a few hours. After dehydration, resin-embedment and polymerization, tissues were sectioned as $70 \mathrm{~nm}$ thin sections and deposited onto holey carbon-coated grids. A final step of quick staining with uranyl acetate and lead citrate was performed to enhance the tissue electronic contrast. Close inspection of the $1 \mathrm{~mm} \times 1 \mathrm{~mm}$ section allowed us to find well-assembled iron oxide nanoparticles whose $150 \mathrm{~nm}$ wide geometry was reminiscent of the arrangement and dimension of the injected bimodal nanoassemblies. Several other nanoparticles adopted a little looser organization, though still featuring a circular layout (Fig. 7 and see ESI Fig. S8†).

The presence of iron-enriched ferritin agglomerates was ruled out as these latter are usually observed with fivefold higher concentrations of magnetite NP suspensions at day 14 post injection. ${ }^{48}$ Our observations were observed $24 \mathrm{~h}$ after injection, which is insufficient to recruit enough ferritin proteins and create aggregates as large as those observed. TEM investigations confirm the highly cohesive character of the selfassembled magnetic and fluorescent units constituting the reverse core-shell architectures of fluo@mag@PAA. They point out the benefit to use such a kind of nanoassembly for crosscorrelated imaging spanning the millimeter, micrometer and nanometer scales.

\section{Conclusions}

In summary, we have synthesized hydrophilic bimodal nanoassemblies fluo@mag@PAA, based on an innovative "raspberry" design, displaying superparamagnetic nanoparticles complexed at the surface of a matrix-free hydrophobic fluorescent organic core. These nanoassemblies exhibit large colloidal stability in alcoholic solvents and aqueous media over a broad range of $\mathrm{pH}$ and ionic strengths, as required for any investigation in physiological media The great compactness of the iron oxide nanoparticle shell and the absence of continuous coating led to constructive transverse relaxivities that appear to be remarkable for $7 \mathrm{~nm}$ wide maghemite nanoparticles. MRI imaging could be performed under normal conditions using doses of magnetic material ten times smaller than those commonly reported. A clear $-49 \%$ dark enhancement after injection in small rodents and accumulation of fluo@mag@PAA in the liver was measured, in agreement with the $T_{2}$ contrast properties of superparamagnetic nano-objects. The stiff cohesion and colloidal stabilization brought by the external polyelectrolyte shell avoids aggregation as well as disintegration of the nanoassembly following in vivo injection. The high oneand two-photon brightness up to $10^{7} \mathrm{~mol}^{-1} \mathrm{~L} \mathrm{~cm}^{-1}$ and $3.7 \times$ $10^{5} \mathrm{GM}$, respectively, enables easy visualization of the nanoassemblies in highly pigmented organs like the liver. TEM imaging of the sectioned liver evidenced the structural integrity of the nanoassembly $24 \mathrm{~h}$ after in vivo injection, making the multiscale and multimodal assignment possible. Biodegradation and bioconjugation of these innovative platforms with appropriate chemical and biological entities are currently performed. This would open new prospects to unambiguously follow the biodistribution of administrated drugs or stem cells after appropriate internalization of the nanoassemblies, that is often speculated due to the lack of sensitive and stable enough imaging agents.

\section{Experimental section}

\section{Nanoassembly preparation}

A solution of fluorescent phosphonic compound ${ }^{22}$ dissolved in THF (50 $\mu \mathrm{L}, 0.1 \mathrm{wt} \%$ ) was added under vigorous stirring to a solution of maghemite nanoparticles in nitric acid $(2.5 \mathrm{~mL}$, $0.006 \mathrm{wt} \%, \mathrm{pH}=1.2$ ). After a few seconds, the magnetofluorescent nanoassemblies fluo@mag were formed. Polyacrylic acid $(2.1 \mathrm{kDa}, 5 \mathrm{mg})$ was added as powder; ammonium hydroxide $\left(1 \mathrm{~mol} \mathrm{~L}^{-1}\right)$ was added dropwise under stirring until $\mathrm{pH}=9$ was reached. The resulting translucent solution was allowed to stir for further $30 \mathrm{~min}$ and dialyzed using a Spectra Por membrane (Standard Grade Regenerated Cellulose; cut-off: 8-10 kDa) against Millipore water $(600 \mathrm{~mL})$ over $24 \mathrm{~h}$ until the final pH of the fluo@mag@PAA nanoassembly suspensions reached a value of 7 .

\section{Lyophilization procedure for long-term storage and redispersion procedure}

In order to store the resulting fluo@mag@PAA nanoassemblies over a long period of time in the solid state, volumes from 1 to 3 $\mathrm{mL}$ of nanoassembly suspensions were added to a glass vial such that the height of the liquid was not higher than $1 \mathrm{~cm}$. The suspension was allowed to freeze in a bath of liquid nitrogen. Lyophilization was performed over $9 \mathrm{~h}$ to $12 \mathrm{~h}$ to yield a dark red powder that was stored at $-18{ }^{\circ} \mathrm{C}$. Nanoassembly redispersion was easily performed by adding the suitable solvent (water, physiological media, alcoholic solvents such as ethanol) to the lyophilized sample. No ultrasound treatment was required since the nanoassemblies undergo no aggregation upon redispersion. Iron concentration in the range $0.6-2 \times 10^{-3} \mathrm{~mol} \mathrm{~L}^{-1}$ could be obtained following the added amount of solvent.

\section{Nanoparticle structural characterizations}

The nanoassembly morphology was investigated by transmission electron microscopy (model Hitachi HF2000-FEG or 
JEOL JEM-1230S). Suspensions of nanoassemblies were deposited onto holey carbon-coated copper grids (300 mesh) purchased from Delta Technologies. The hydrodynamic diameter and size dispersion of nanoparticles were determined by dynamic light scattering (DLS) by using a nanoparticle size analyzer Zetasizer Nano ZS ZEN 3600 (Malvern Instruments) equipped with a $4 \mathrm{~mW}$ He-Ne laser, operating at $633 \mathrm{~nm}$, and a photomultiplier detector collecting backscattered light at an angle of $175^{\circ}$. Measurements of aqueous suspensions of nanoassemblies were carried out at $25{ }^{\circ} \mathrm{C}$. For each sample, intensity measurements were carried out in a multi-acquisition mode implying automatically adjusted correlograms, and averaged measurements on 3 acquisitions. The nanoassembly mean size and distribution width were obtained by fitting each correlogram with a cumulants algorithm. Measurements of surface potential $\zeta$ were carried out by using a Zetasizer Nano ZS ZEN 3600 (Malvern). The samples were placed in plastic cells. Several measurements were realized for each sample according to a predefined operating procedure.

\section{Magnetic relaxometry}

Measurements of ${ }^{1} \mathrm{H}$ NMR longitudinal $\left(T_{1}\right)$ and transverse $\left(T_{2}\right)$ relaxation times at different frequencies $\nu$ were performed at room temperature on water diluted solutions of samples, fluo@mag@PAA nanoassemblies, and free iron oxide nanoparticles coated with PAA (mag@PAA) in the range $10 \mathrm{kHz} \leq \nu \leq$ $60 \mathrm{MHz}$ for $T_{1}$ and $15 \mathrm{MHz} \leq \nu \leq 60 \mathrm{MHz}$ for $T_{2}$. Generation and detection of the NMR signal involved a Smartracer Stelar relaxometer (using the Fast-Field-Cycling technique) for $10 \mathrm{kHz}$ $\leq \nu \leq 10 \mathrm{MHz}$, a Stelar Spinmaster spectrometer for $\nu>10 \mathrm{MHz}$. In the second case, the standard radio frequency excitation sequences Carr-Purcell-Meiboom-Gill (CPMG)-like $\left(T_{2}\right)$ and saturation recovery $\left(T_{1}\right)$ were used. The efficiency of samples as MRI contrast agents was determined from the measured $\mathrm{T}_{1}$ and $\mathrm{T}_{2}$ by calculating the nuclear longitudinal $r_{1}$ and transverse $r_{2}$ relaxivities as given by $r_{\mathrm{i}}=\left[\left(1 / T_{\mathrm{i}}\right)-\left(1 / T_{\mathrm{i}}\right)_{\text {water }}\right] / c$, where $\left(1 / T_{\mathrm{i}}\right)$ is the measured value for the sample, $c$ is the concentration of magnetic centers (in mmol L $\left.{ }^{-1}\right)$ and $\left(1 / T_{\mathrm{i}}\right)_{\text {water }}$ refers to the nuclear relaxation rate of diamagnetic water molecules. $r_{1}$ and $r_{2}$ relaxivities measure the increase of the nuclear spin-lattice and spin-spin relaxation rates respectively, due to the presence of the magnetic system in the solvent.

\section{Photophysical measurements}

UV-visible absorption spectra were recorded using a Varian Model Cary 5E spectrophotometer, using an integrating sphere DRA 2500. Corrected emission spectra were obtained using a Jobin-Yvon. Inc spectrofluorimeter (Fluorolog 2). Fluorescence quantum yields in solution were determined by using Coumarine $540 \mathrm{~A}$ in ethanol as a fluorescence standard $\left(\Phi_{\mathrm{f}}=0.38\right)$.

\section{Two-photon absorption cross section measurements}

Characterization of the two-photon absorption properties of each compound was determined using two-photon excited fluorescence (TPEF) measurements. The source consisted of a tunable Ti:sapphire laser source delivering $\approx 100 \mathrm{fs}$ pulses at a
$76 \mathrm{MHz}$ repetition rate over the spectral range 770 to $940 \mathrm{~nm}$ (Tsunami - Spectra Physics). Intensities of the TPEF signals of each sample were measured relative to those of a $3 \times 10^{-5}$ molL $^{-1}$ solution of fluorescein in $0.01 \mathrm{~mol} \mathrm{~L}^{-1}$ sodium hydroxide solution $(\mathrm{pH}=12)$. Calculations of the ratio of both emission signals provide further determination of the twophoton absorption cross-section of the unknown sample $\left(\sigma_{\text {TPA }}^{\text {s }}\right)$, assuming equal one- and two-photon fluorescence quantum yields: ${ }^{49}$

$$
\sigma_{\mathrm{TPA}}^{\mathrm{s}}=\frac{\varphi_{\mathrm{F}}^{\mathrm{ref}} c_{\mathrm{ref}} I_{\mathrm{s}}}{\varphi_{\mathrm{F}}^{\mathrm{s}} c_{\mathrm{s}} I_{\mathrm{ref}}} \sigma_{\mathrm{TPA}}^{\mathrm{ref}}
$$

where $\varphi_{\mathrm{F}}, c, I$ represent respectively the fluorescence quantum yield, the concentration (inferred from UV-vis absorption measurements in solution), and the total fluorescence signal measured using a Channel Plate Multiplier detector (Perkin MP993). The $s$ and ref superscripts respectively stand for the unknown sample and the fluorescein reference. The quadratic dependence of the TPEF intensity with the power of the incident exciting infrared beam was systematically checked. Spectral matching between the one-photon and two-photon emission spectra was also verified.

\section{MRI imaging on small rodents}

Animal experiments were performed in accordance with the INSERM animal protection guidelines and approved by local governmental authorities. Experiments were carried out on five female BALB/c mice (5 week old) (Iffa Credo, L'Arbresle, France), weighing between 25 and $31 \mathrm{~g}$. Animals were anaesthetized using isoflurane in order to stabilize the heart rate at approximately 30 heartbeats per minute. A suspension of fluo@ mag@PAA nanoassemblies in HBSs buffer $(250 \mu \mathrm{L}, 1.3 \mu \mathrm{mol}$ $\mathrm{mL}^{-1}$ Fe concentration) was injected in the mouse caudal vein. MRI experiments were performed with a dedicated smallanimal 4.7 Tesla MR system (Biospec 47/40 USR Bruker), using a quadrature transmit/receive body coil with a $35 \mathrm{~mm}$ inner diameter. Mice were imaged twice: before and after a 25-30 min delay after injection, under general anesthesia with isoflurane gas. A $T_{2}{ }^{*}$-weighted gradient echo sequence (FLASH) was used with the following parameters: repetition time $\mathrm{TR}=300 \mathrm{~ms}$, echo time $\mathrm{TE}=5 \mathrm{~ms}$, flip angle $30^{\circ}$, field of view $4 \mathrm{~cm} \times 4 \mathrm{~cm}$, matrix $256 \times 256$, resolution $156 \mu \mathrm{m} \times 156 \mu \mathrm{m}$, average number 8, and 14 axial slices. Regions of interest (ROI) were defined in the spleen and in the liver by using the freeware image processing tool ImageJ. The signal intensity of the ROI was normalized by the signal from a water phantom placed next to the mouse into the coil. Enhancement (ENH) between normalized signals of imaged animals before $\left(\mathrm{SI}_{\text {ref }}\right)$ and after injection (SI) was calculated in each ROI as ENH (\%) = $\left(\mathrm{SI}-\mathrm{SI}_{\mathrm{ref}}\right)-100 / \mathrm{SI}_{\mathrm{ref}}$.

\section{One- and two-photon fluorescence microscopy}

One-photon fluorescence imaging was performed using a Nikon A1R Si microscope, equipped with $\mathrm{Ar}^{+}$and $\mathrm{He}-\mathrm{Ne}$ lasers as excitation sources, an oil-immersion objective Plan Apo, $60 \times$, $\mathrm{NA}=1.4, \lambda_{\text {exc }}=488 \mathrm{~nm}$. Two-photon fluorescence imaging was 
performed using a Zeiss LSM 780 Axio Observer microscope, equipped with a tunable mode-locked Ti:sapphire femtosecond laser (Mai Tai DeepSee, Spectra-Physics 690-1060 nm), a 32channel QUASAR GaAsP detector array allowing for spectral imaging, and a water immersion objective C-Apochromat $(63 \times$ $\mathrm{NA}=1.15)$. The samples were excited with $1 \%$ of the $830 \mathrm{~nm}$ light $(2.86 \mathrm{~W}$ maximum power at $830 \mathrm{~nm})$ and their emission spectra were observed either as 32 separate spectral channels with $\sim 10 \mathrm{~nm}$ intervals covering a total range of 411-758 nm (lambda mode) or as 2 channels combining the detectors in the range 411-490 and 550-758 $\mathrm{nm}$, respectively (channel mode). All image acquisition was performed using the Zeiss Zen2012 software.

\section{Acknowledgements}

Carole La and Antoine Bezos from LPGN-CNRS 6112 (Nantes) and Gwenhael Autrey from PARCC-Centre Pompidou (Paris) are strongly acknowledged for their great help in ICP-OES titrations and MRI experiments, respectively. The Région Pays de la Loire, France, is gratefully acknowledged for its strong financial support. The Campus France is acknowledged for its support through the PHC France-Belgium Tournesol 2013 joint project MONSABIC. Pieter Vanden Berghe from the Cell Imaging Core (CIC-KULeuven) is warmly thanked for giving access to twophoton microscopy imaging facilities. Paolo Arosio from Università degli Studi di Milano and Prof. Maurizio Corti from Università di Pavia are thanked for their scientific and technical support in NMR measurements.

\section{Notes and references}

1 J. Kim, Y. Piao and T. Hyeon, Chem. Soc. Rev., 2009, 38, 372390.

2 Z. Cheng, A. Al Zaki, J. Z. Hui, V. R. Muzykantov and A. Tsourkas, Science, 2012, 338, 903-910.

3 J. V. Jokerst and S. S. Gambhir, Acc. Chem. Res., 2011, 44, 1050-1060.

4 E. Boisselier and D. Astruc, Chem. Soc. Rev., 2009, 38, 17591782.

5 J. Cheon and J. H. Lee, Acc. Chem. Res., 2008, 41, 1630-1640. 6 D.-E. Lee, H. Koo, I.-C. Sun, J. H. Ryu, K. Kim and I. C. Kwon, Chem. Soc. Rev., 2012, 41, 2656-2672.

7 M. Vendrell, D. T. Zhai, J. C. Er and Y. T. Chang, Chem. Rev., 2012, 112, 4391-4420.

8 M. Sauer, J. Hofkens and J. Enderlein, Handbook of Fluorescence Spectroscopy and Imaging, Wiley-VCH Weinheim, 2011.

9 P. Caravan, Acc. Chem. Res., 2009, 42, 851-862.

10 S. Viswanathan, Z. Kovacs, K. N. Green, S. J. Ratnakar and A. D. Sherry, Chem. Rev., 2010, 110, 2960-3018.

11 R. Duncan and R. Gaspar, Mol. Pharmaceutics, 2011, 8, 21012141.

12 S. Santra and A. Malhotra, Wiley Interdiscip. Rev.: Nanomed. Nanobiotechnol., 2011, 3, 501-510.

13 S. Laurent, D. Forge, M. Port, A. Roch, C. Robic, L. Vander Elst and R. N. Muller, Chem. Rev., 2008, 108, 2064-2110.
14 N. C. Bigall, W. J. Parak and D. Dorfs, Nano Today, 2012, 7, 282-296.

15 J.-H. Lee, Y.-W. Jun, S.-I. Yeon, J.-S. Shin and J. Cheon, Angew. Chem., Int. Ed., 2006, 45, 8160-8162.

16 F. Bertorelle, C. Wilhelm, J. Roger, F. Gazeau, C. Ménager and V. Cabuil, Langmuir, 2006, 22, 5385-5391.

17 V. Salgueiriño-Maceira, M. A. Correa-Duarte, M. Spasova, L. M. Liz-Marzán and M. Farle, Adv. Funct. Mater., 2006, 16, 509-514.

18 N. Insin, J. B. Tracy, H. Lee, J. P. Zimmer, R. M. Westervelt and M. G. Bawendi, ACS Nano, 2008, 2, 197-202.

19 A. Quarta, R. Di Corato, L. Manna, S. Argentiere, R. Cingolani, G. Barbarella and T. Pellegrino, J. Am. Chem. Soc., 2008, 130, 10545-10555.

20 P.-W. Lee, S.-H. Hsu, J.-J. Wang, J.-S. Tsai, K.-J. Lin, S.-P. Wey, F.-R. Chen, C.-H. Lai, T.-C. Yen and H.-W. Sung, Biomaterials, 2010, 1316-1324.

21 K. J. C. van Bommel, A. Friggeri and S. Shinkai, Angew. Chem., Int. Ed., 2003, 42, 980-999.

22 A. Faucon, J. Fresnais, A. Brosseau, P. Hulin, S. Nedellec, J. Hemez and E. Ishow, J. Mater. Chem. C, 2013, 1, 3879-3886.

23 J. Y. Kim, O. Voznyy, D. Zhitomirsky and E. H. Sargent, Adv. Mater., 2013, 25, 5742-5749.

24 C. Wu and D. T. Chiu, Angew. Chem., Int. Ed., 2013, 52, 30863109.

25 A. Kaeser and A. P. H. J. Schenning, Adv. Mater., 2010, 22, 2985-2997.

26 S. Fery-Forgues, Nanoscale, 2013, 51, 8428-8437.

27 Q. H. Cui, Y. S. Zhao and J. Yao, Adv. Mater., 2014, 24, 23322336.

28 T. Torres and G. Bottari, Organic Nanomaterials: Synthesis, Characterization, and Device Applications, John Wiley \& Sons, Inc., Hoboken, New Jersey, 2013.

29 R. J. Hickey, J. Koski, X. Meng, R. A. Riggleman, P. J. Zhang and S. J. Park, ACS Nano, 2014, 8, 495-502.

30 D. Ling, W. Park, S.-j. Park, Y. Lu, K. S. Kim, M. J. Hackett, B. H. Kim, H. Yim, Y. S. Jeon, K. Na and T. Hyeon, J. Am. Chem. Soc., 2014, 136, 5647-5655.

31 H.-H. Fang, J. Yang, J. Feng, T. Yamao, S. Hotta and H.-B. Sun, Laser Photonics Rev., 2014, 8, 687-715.

32 Y. S. Zhao, H. Fu, F. Hu, A. Peng, W. Yang and J. Yao, Adv. Mater., 2008, 20, 79-83.

33 J. Zhang, Q. Zou and H. Tian, Adv. Mater., 2013, 25, 378-399. 34 K. Petkau, A. Kaeser, I. Fischer, L. Brunsveld and A. P. H. J. Schenning, J. Am. Chem. Soc., 2011, 133, 1706317071.

35 J. S. Basuki, A. Jacquemin, L. Esser, C. Boyer and R. M. Davis, Polym. Chem., 2014, 5, 2611-2620.

36 M. F. Casula, P. Floris, C. Innocenti, A. Lascialfari, M. Marinone, M. Corti, R. A. Sperling, W. J. Parak and C. Sangregorio, Chem. Mater., 2010, 22, 1739-1748.

37 M. S. Martina, J. P. Fortin, C. Menager, O. Clement, G. Barratt, C. Grabielle-Madelmont, F. Gazeau, V. Cabuil and S. Lesieur, J. Am. Chem. Soc., 2005, 127, 10676-10685.

38 P. Arosio, J. Thevenot, T. Orlando, F. Orsini, M. Corti, M. Mariani, L. Bordonali, C. Innocenti, C. Sangregorio, 
H. Oliveira, S. Lecommandoux, A. Lascialfari and O. Sandre, J. Mater. Chem. B, 2013, 1, 5317-5328.

39 A. Kostopoulou, S. K. P. Velu, K. Thangavel, F. Orsini, K. Brintakis, S. Psycharakis, A. Ranella, L. Bordonali, A. Lappas and A. Lascialfari, Dalton Trans., 2014, 43, 83958404.

40 G. Béalle, R. Di Corato, J. Kolosnjaj-Tabi, V. Dupuis, O. Clément, F. Gazeau, C. Wilhelm and C. Ménager, Langmuir, 2012, 28, 11834-11842.

41 G. S. He, L. S. Tan, Q. Zheng and P. N. Prasad, Chem. Rev., 2008, 108, 1245-1330.

42 M. Pawlicki, H. A. Collins, R. G. Denning and H. L. Anderson, Angew. Chem., Int. Ed., 2009, 48, 3244-3266. 43 A. Gaiduk, M. Yorulmaz, E. Ishow and M. Orrit, ChemPhysChem, 2012, 13, 946-951.
44 E. Ishow, A. Brosseau, G. Clavier, K. Nakatani, P. Tauc, C. Fiorini-Debuisschert, S. Neveu, O. Sandre and A. Leaustic, Chem. Mater., 2008, 20, 6597-6599.

45 V. Parthasarathy, S. Fery-Forgues, E. Campioli, G. Recher, F. Terenziani and M. Blanchard-Desce, Small, 2011, 7, 3219-3229.

46 M. Breton, G. Prevel, J. F. Audibert, R. Pansu, P. Tauc, B. Le Pioufle, O. Francais, J. Fresnais, J. F. Berret and E. Ishow, Phys. Chem. Chem. Phys., 2011, 13, 13268-13276.

47 M. Y. Berezin and S. Achilefu, Chem. Rev., 2010, 110, 26412684.

48 L. Lartigue, D. Alloyeau, J. Kolosnjaj-Tabi, Y. Javed, P. Guardia, A. Riedinger, C. Péchoux, T. Pellegrino, C. Wilhelm and F. Gazeau, ACS Nano, 2013, 7, 3939-3952.

49 M. A. Albota, C. Xu and W. W. Webb, Appl. Opt., 1998, 37, 7352-7356. 